\title{
Role of Caspase-11 Non-Canonical Inflammasome in Macrophage-Mediated Inflammatory Responses
}

\author{
Young-Su Yi ${ }^{*}$
}

Department of Pharmaceutical and Biomedical Engineering, Cheongju University, Cheongju 28503, South Korea

*Corresponding author: Young-Su Yi, Ph.D., Department of Pharmaceutical and Biomedical Engineering, Cheongju University, 298 Daesung-Ro, Cheongwon-Gu, Cheongju-Si, Chungcheongbuk-Do 28503, South Korea, Tel: +82-43-2297852, Fax: $+82-43-229-8577$

\begin{abstract}
Inflammation is a complex biological response mediated by macrophages to protect the body from the pathogens and danger signals. The inflammatory response is initiated by priming, a process increasing the expression of inflammatory genes by extracellular pattern-recognition receptor (PRR)mediated detection of pathogens, followed by triggering, a process detecting cytosolic pathogens by intracellular PRRs. Triggering induces the formation of intracellular PRR complexes called inflammasomes composed of two main groups; canonical and non-canonical inflammasomes. Unlike canonical inflammasomes, non-canonical inflammasomes were recently discovered, and the knowledge of the roles of non-canonical inflammasomes in inflammatory responses and human diseases is not still enough. Mouse caspase- 11 and human caspase- $4 / 5$ were identified as non-canonical inflammasomes, and many efforts have been made to demonstrate the regulatory functions of these non-canonical inflammasomes in inflammatory responses and several human diseases. This review discusses the recent research progress to understand the roles of the caspase-11 non-canonical inflammasome in macrophagemediated inflammatory responses, which can provide the insight and contribute to developing potential diagnostic and therapeutic agents to prevent and treat human infectious and inflammatory/autoimmune diseases.
\end{abstract}

\section{Keywords}

Caspase-11, Non-canonical inflammasome, Inflammatory response, Macrophage

\section{Abbreviations}

PRRs: Pattern Recognition Receptors; PAMPs: Pathogenassociated Molecular Patterns; DAMPs: Danger-associated Molecular Patterns; NF-kB: Nuclear Factor-Kappa B; AP1: Activator Protein-1; IRFs: Interferon Regulatory Factors; NLRs: Nucleotide-binding Oligomerization Domain-Like Receptors; RLRs: RIG-I-like Receptors; AIM2: Absent in
Melanoma 2; ALRs: AIM2-like Receptors; GSDMD: Gasdermin D; PYD: Pyrin Domain; NACHT: Nucleotidebinding and Oligomerization Domain; LRRs: Leucine-Rich Repeats; FIND: Functional-to-find Domain; CARD: Caspase Recruit Domain; HIN200: Hematopoietic InterferonInducible Nuclear Protein 200; LPS: Lipopolysaccharide; IL: Interleukin; OMV: Outer Membrane Vesicle

\section{Introduction}

Inflammation is an innate immune response to protect the body from the invasion of pathogens and intracellular danger signals, and the hallmarks of inflammation are redness, swelling, pain, heat, and loss of tissue functions [1-3]. Inflammatory response is mediated by innate immune cells, such as M1 macrophages with two consecutive steps; priming and triggering. Priming is initiated via recognizing pathogenassociated molecular patterns (PAMPs) and dangerassociated molecular patterns (DAMPs) by patternrecognition receptors (PRRs), such as Toll-like receptors expressed on macrophages and subsequently induces inflammatory signaling cascades, such as nuclear factor-kappa B (NF-kB), activator protein-1 (AP-1), and interferon regulatory factors (IRFs), leading to the overexpression of inflammatory genes [4-6]. On the other hand, some PRRs, such as nucleotide-binding oligomerization domain-like receptors (NLRs), RIG-Ilike receptors (RLRs), absent in melanoma 2 (AIM2)like receptors (ALRs), and caspase- 11 are localized in the cytosol of macrophages and induce inflammatory responses by detecting cytosolic PAMPs and DAMPs [712]. Triggering is initiated by recognizing the cytosolic

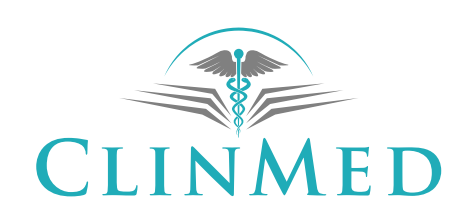

INTERNATIONAL LIBRARY

Citation: Young-Su Y (2018) Role of Caspase-11 Non-Canonical Inflammasome in Macrophage-Mediated Inflammatory Responses. Int J Immunol Immunother 5:034. doi.org/10.23937/2378-3672/1410034 Accepted: December 12, 2018: Published: December 14, 2018

Copyright: (C 2018 Young-Su Y. This is an open-access article distributed under the terms of the Creative Commons Attribution License, which permits unrestricted use, distribution, and reproduction in any medium, provided the original author and source are credited. 
ligands, and these intracellular PRRs are activated by assembling a large protein complex, known as an inflammasome. Inflammasomes are classified into two groups; canonical inflammasomes, such as NLR family and AIM2 inflammasomes and noncanonical inflammasomes, such as mouse caspase-11 and human caspase- 4 and -5 inflammasomes [7-12]. Inflammasomes are activated in response to the ligands specific for each inflammasome, and inflammasome activation induces 1) Gasdermin D (GSDMD) processing and GSDMD-mediated pyroptosis by generating GSDMD pores, an inflammatory form of cell death and 2) Proteolytic activation of pro-caspase-1 and active caspase-1-mediated maturation and secretion of proinflammatory cytokines, interleukin (IL)-1 $\beta$ and IL-18 through the GSDMD pores [7-12].

Canonical inflammasomes have long been investigated in inflammatory conditions and several human diseases, by contrast, non-canonical inflammasomes, such as mouse caspase- 11 and human caspase- $4 / 5$ were recently discovered. However, although the knowledge of the functional roles of these non-canonical inflammasomes is limited, a number of emerging studies have successfully demonstrated the regulatory roles of caspase-11 non-canonical inflammasomes in inflammatory responses and human diseases. This review aimed to summarize and discuss the recent study progress in understanding the regulatory roles of the caspase-11 non-canonical inflammasome in macrophage-mediated inflammatory responses and to provide the insight for the development of promising drugs to prevent and treat various infectious and inflammatory/autoimmune diseases.

\section{Structure and Activation of Inflammasomes}

Inflammasomes are classified into two groups; canonical and non-canonical inflammasomes. There are several types of canonical inflammasomes, such as NLR family inflammasomes, including NLRP1, NLRP3, and NLRC4 and non-NLR family inflammasome, including
A

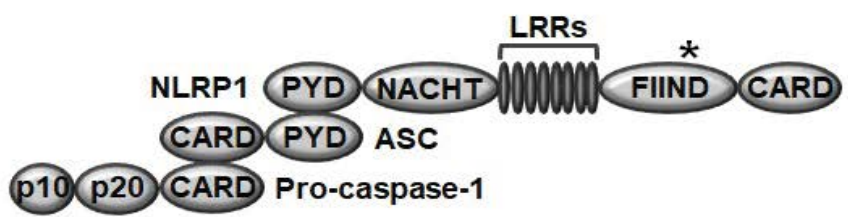

C

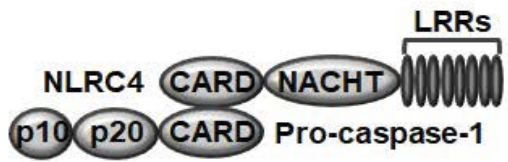

E

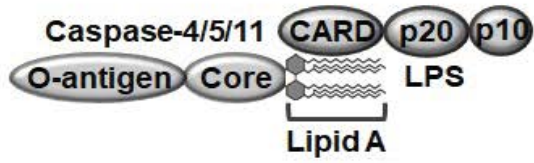

B

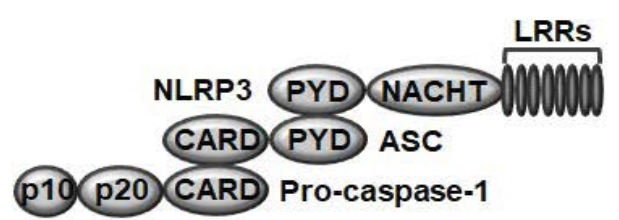

D

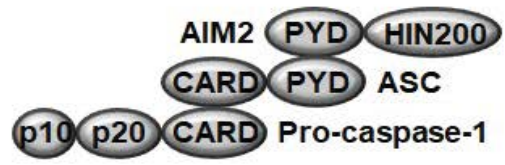

$\mathbf{F}$

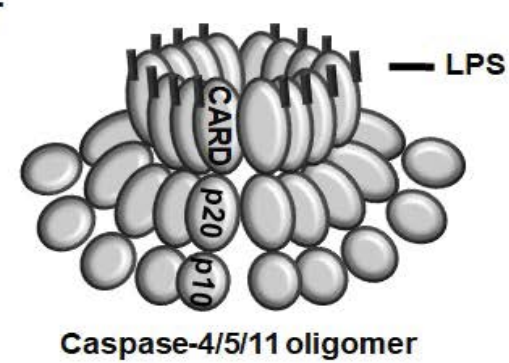

Figure 1: The structure of canonical and non-canonical inflammasomes A) NLRP1 consisting of a PYD, a NACHT, LRRs, a FIIND, and a CARD assembles inflammasome by the interaction of NLRP1 with ASC through their PYDs and the subsequent interaction with pro-caspase-1 through their CARDs; B) NLRP3 consisting of a PYD, a NACHT, and LRRs assembles inflammasome by the interaction of NLRP3 with ASC through their PYDs and the subsequent interaction with pro-caspase-1 through their CARDs; C) NLRC4 consisting of a CARD, a NACHT, and LRRs assembles inflammasome by the direct interaction of NLRC4 and pro-caspase-1 through their CARDs; D) AIM2 consisting of a PYD and a HIN200 domain assembles inflammasome by the interaction of AIM2 and ASC through their PYDs and subsequent interaction with pro-caspase- 1 through their CARDs; E) Mouse caspase- 11 and human caspase-4/5 consisting of a CARD, a p20, and a p10 assemble inflammasomes by the direct interaction of caspase-4/5/11 CARDs and the lipid A moiety of intracellular LPS; F) Once caspase-4/5/11 bind with intracellular LPS, LPS-caspase-4/5/11 complexes are oligomerized and activated.

NLR: Nucleotide-binding oligomerization domain-like receptor; PYD: Pyrin domain; NACHT: Nucleotide-binding and oligomerization domain; LRRs: Leucine-rich repeats; FIIND: Function to find domain; CARD: Caspase recruit domain; Caspase: Cysteine-aspartic protease; AIM2: Absent in melanoma 2; HIN: Hematopoietic interferon-inducible nuclear proteins; Caspase: Cysteine-aspartic protease; LPS: Lipopolysaccharide.

*: Autocatalytic cleavage site. 
AIM2 inflammasome. NLRP1 consisting of a pyrin domain (PYD), a nucleotide-binding and oligomerization domain (NACHT), leucine-rich repeats (LRRs), a functionalto-find domain (FIIND), and a caspase recruit domain (CARD) assembles a NLRP1 inflammasome in response to Bacillus anthracis lethal toxin by interacting NLRP1, ASC, a bipartite adaptor, and pro-caspase-1 through their PYDs and CARDs (Figure 1A) [9-11,13]. NLRP3 consisting of a PYD, a NACHT, and LRRs assembles a NLRP3 inflammasome in response to various ligands, including pathogen nucleic acid hybrids, bacterial poregenerating toxins, extracellular ATP, monosodium urate, cholesterol, potassium efflux, silica, crystals, alum, asbestos, $\beta$-amyloids, oxidized mitochondrial DNA, mitochondrial ROS, and hyaluronan by interacting NLRP3, ASC, and pro-caspase-1 through their PYDs and CARDs (Figure 1B) [9-11,13]. NLRC4 consisting of a CARD, a NACHT, and LRRs assembles NLRC4 inflammasome in response to bacterial needle subunits, flagellin, and type III secretion apparatus by directly interacting NLRC4 and ASC through their CARDs (Figure 1C) [9-11,13]. AIM2, an interferon-inducible p200 protein family is a non-NLR family member and was initially identified as a sensor of intracellular double-stranded DNA originated from the invading pathogens $[14,15]$. AIM2 consisting of a PYD and a hematopoietic interferon-inducible nuclear protein 200 (HIN200) assembles AIM2 inflammasome in response to intracellular pathogen double-stranded DNA by interacting AIM2, ASC, and pro-caspase-1 through their PYDs and CARDs (Figure 1D) [9-11,13].

Several types of non-canonical inflammasomes have also been discovered. Caspase-11 consisting of a CARD, p20, and p20 assembles caspase-11 inflammasome in response to intracellular lipopolysaccharide (LPS) derived from the gram-negative bacteria by the direct interaction between the CARD motif of caspase-11 and the lipid A moiety of intracellular LPS (Figure 1E) [9-11,13]. Caspase-11 was identified in mice, and interestingly, caspase- 4 and -5 were identified as human homologs of mouse caspase-11 [16-18]. Caspase-4/5 have a similar structure with caspase-11 consisting of a CARD, p20, and p10 and also assemble caspase-4/5 inflammasomes in response to gram-negative bacterium-originated intracellular LPS in a same way of caspase-11 (Figure 1E) [9-11,13]. Once caspase-4/5/11 interact with intracellular LPS to form caspase-4/5/11 non-canonical inflammasomes, LPS-caspase-4/5/11 complexes are subsequently oligomerized by the direct interaction between CARDs of caspase-4/5/11, leading to the activation of caspase-4/5/11 inflammasomes (Figure 1F). The structure and ligands of inflammasomes are summarized in Table 1.

\section{Role of Caspase-11 Non-canonical Inflammas-} ome in Macrophage-mediated Inflammatory Responses

Previous studies have successfully demonstrated that inflammasomes are activated by a variety of ligands, present in innate immune cells, such as M1 macrophages during inflammatory responses. Interestingly, unexpected observation was reported that cholera toxin B-induced inflammasome activation and inflammatory responses were abolished in the bone marrow-derived macrophages isolated from the 12956 mouse strain which lacks functional caspase-11 proteins due to the polymorphism in the caspase-11 gene locus and that septic shock induced by the lethal dose of LPS was much less in these mice [19]. This observation suggests that caspase-11 is not a member of canonical inflammasomes and plays a unique role during macrophage-mediated inflammatory responses in a canonical inflammasome-independent manner, therefore, the caspase-11 inflammasome was named as non-canonical inflammasome.

As discussed earlier, caspase-11 non-canonical inflammasome is activated by intracellular LPS derived

Table 1: Summary of inflammasome structure and ligands.

\begin{tabular}{|c|c|c|c|c|}
\hline Class & Inflammasomes & $\begin{array}{l}\text { Inflammasome } \\
\text { composition }\end{array}$ & PRR structure (N to $\mathrm{C}$ ) & Ligands \\
\hline \multirow{4}{*}{$\begin{array}{l}\text { Canonical } \\
\text { Inflammasomes }\end{array}$} & $\begin{array}{l}\text { NLRP1 } \\
\text { Inflammasome }\end{array}$ & $\begin{array}{l}\text { hNLRP1-(P)-ASC-(C)-pro- } \\
\text { caspase-1 }\end{array}$ & $\begin{array}{l}\text { PYD-NACHT-LRRs-FIIND- } \\
\text { CARD }\end{array}$ & Bacillus anthracis lethal toxin \\
\hline & $\begin{array}{l}\text { NLRP3 } \\
\text { Inflammasome }\end{array}$ & $\begin{array}{l}\text { NLRP3-(P)-ASC-(C)-pro- } \\
\text { caspase-1 }\end{array}$ & PYD-NACHT-LRRs & $\begin{array}{l}\text { Pathogen nucleic acid hybrids, } \\
\text { Bacterial pore-generating toxins, } \\
\text { Extracellular ATP, Oxidized } \\
\text { mtDNA, MtROS, Monosodium } \\
\text { urate, Cholesterol, Potassium } \\
\text { efflux, Silica, Crystals, Alum, } \\
\text { Asbestos, } \beta \text {-amyloids, Hyaluronan }\end{array}$ \\
\hline & $\begin{array}{l}\text { NLRC4 } \\
\text { Inflammasome }\end{array}$ & NLRC4-(C)-pro-caspase-1 & CARD-NACHT-LRRs & $\begin{array}{l}\text { Bacterial needle subunits, Flagellin, } \\
\text { Type III secretion apparatus }\end{array}$ \\
\hline & $\begin{array}{l}\text { AIM2 } \\
\text { Inflammasome }\end{array}$ & $\begin{array}{l}\text { AIM2-(P)-ASC-(C)-pro- } \\
\text { caspase-1 }\end{array}$ & PYD-HIN200 & Microbial double-stranded DNA \\
\hline $\begin{array}{l}\text { Non-canonical } \\
\text { Inflammasomes }\end{array}$ & $\begin{array}{l}\text { Caspase-4/5/11 } \\
\text { Inflammasome }\end{array}$ & Caspase-4/5/11-(C)-LPS & CARD-p20-p10 & Intracellular LPS \\
\hline
\end{tabular}

P) Interaction through PYD; C) Interaction through CARD. 
from gram-negative bacteria and is not activated by the gram-positive bacteria due to the lack of LPS [20]. Hager, et al. also demonstrated that caspase-11 was activated and induced inflammatory responses in the macrophages transfected with the lysates of gram-negative bacteria, but not those of gram-positive bacteria [21]. These studies strongly indicate that caspase-11 non-canonical inflammasome is activated by recognizing intracellular LPS originated from gram-negative bacteria. However, despite the evidence that caspase-11 recognizes intracellular LPS to be activated, how caspase-11 interacts with intracellular LPS was still under question. Biochemical analysis using recombinant caspase-11 expressed and purified in the gram-negative bacterium, $E$. coli demonstrated that caspase-11 directly interacts with LPS to form a huge caspase-11 multimer [18], and unlike canonical inflammasomes, caspase-11 does not need adaptor molecules, such as ASC for the interaction with its ligand, intracellular LPS. Similar with mouse caspase-11, recombinant caspase-4/5 expressed and purified in $E$. coli directly interacts with $E$. coli-derived LPS, and the caspase- $4 / 5$ multimer was produced, while the caspase-4/5 multimer was not formed in the insect cells [18]. Further studies have demonstrated that lipid A moiety of LPS is a critical determinant and sufficient for the interaction with caspase-4/5/11 and the subsequent activation of caspase-4/5/11 non-canonical inflammasomes in macrophage-mediated inflammatory responses [18,21], and that hexa- and penta-acylated lipid $A$ can bind with caspase-4/5/11 and induce the activation of caspase-4/5/11 non-canonical inflammasomes in macrophages, while tetra-acylated lipid A cannot $[21,22]$. These studies suggest that mouse caspase-11 and human caspase-4/5 directly recognize intracellular LPS derived from the gram-negative bacteria, leading to the activation of caspase-4/5/11 non-canonical inflammasomes in macrophage-mediated inflammatory responses.

Many studies have successfully demonstrated the inflammatory events induced by the activation of caspase-11 non-canonical inflammasome in M1 macrophages. Induction of macrophage-mediated inflammatory responses requires two main steps; priming and triggering. Priming is the process by which PRRs recognize PAMPs of pathogens, resulting in the induction of the inflammatory signaling cascades and gene expression of inflammasome components that are required for inflammasome activation [19,23,24]. Priming, itself, neither activates inflammasome activation signaling nor induces the maturation and activation of the inactive-forms of inflammatory molecules, such as pro-caspase-1, pro-IL-1 $\beta$ and pro-IL-18, and pyroptosis, therefore, the second triggering step is required for initiation of inflammasome activation in macrophages during inflammatory responses. The triggering step is initiated through the direct recognition of intracellular LPS by caspase-11, leading to the formation of LPS- caspase-11 oligomers and the activation of caspase- 11 non-canonical inflammasome $[21,23]$. The activation of caspase-11 non-canonical inflammasome subsequently induces the cleavage of GSDMD at the aspartic acid 276 residue $\left(\mathrm{Asp}^{276}\right)$ and produces $\mathrm{N}$-terminal GSDMD fragments that move to the cell membrane and generate GSDMD pores, leading to the pyroptosis [10-13]. Simultaneously, the activation of caspase-11 non-canonical inflammasome also induces the activation of NLRP3 inflammasome and the proteolytic maturation of inactive pro-caspase- 1 to active caspase-1, an effector of inflammasome activation, followed by the proteolytic maturation of inactive pro-inflammatory cytokines, pro-IL-1 $\beta$ and pro-IL-18 to active IL-1 $\beta$ and IL-18 by caspase-1, leading to the secretion of $\mathrm{IL}-1 \beta$ and $\mathrm{IL}-18$ through the GSDMD pores [10-13]. Molecular mechanisms by which caspase-11 non-canonical inflammasome activates NLRP3 canonical inflammasome is still unclear, and the studies elucidating the crosstalk between these canonical and non-canonical inflammasomes in macrophage-mediated inflammatory responses need to be further investigated. The molecular events of caspase-11 non-canonical inflammasome activation during macrophage-mediated inflammatory responses discussed in this chapter are summarized in Figure 2.

\section{Conclusions and Future Perspectives}

The inflammatory response occurs by two consecutive steps; priming and triggering, and the triggering is induced by the activation of inflammasomes. Although regulatory roles and the underlying molecular mechanisms of canonical inflammasome activation in inflammatory responses and a variety of human diseases have long been studied [7,9,11,25-28], noncanonical inflammasomes, such as mouse caspase-11 and human caspase-4/5 were recently discovered, and emerging studies have demonstrated that unlike canonical inflammasomes, caspase-11 non-canonical inflammasome is activated by direct interaction with intracellular LPS originated from gram-negative bacteria, which induces the GSDMD-mediated pyroptosis of inflamed macrophages and the activation of caspase-1, resulting in the maturation and secretion of pro-inflammatory cytokines, IL-1 $\beta$ and IL-18 through GSDMD pores.

Despite a number of recent studies investigating the roles of caspase-11 non-canonical inflammasome in macrophage-mediated inflammatory responses, further studies demonstrating 1) The regulatory roles of caspase-11 non-canonical inflammasome in the pathogenesis of human diseases, 2) The functional crosstalk between canonical and caspase-11 noncanonical inflammasomes in inflammatory responses, 3) The missing puzzle pieces in the caspase-11 noncanonical inflammasome-activated inflammatory responses, and 4) The development of selective and efficient targeting strategies to modulate the activation 


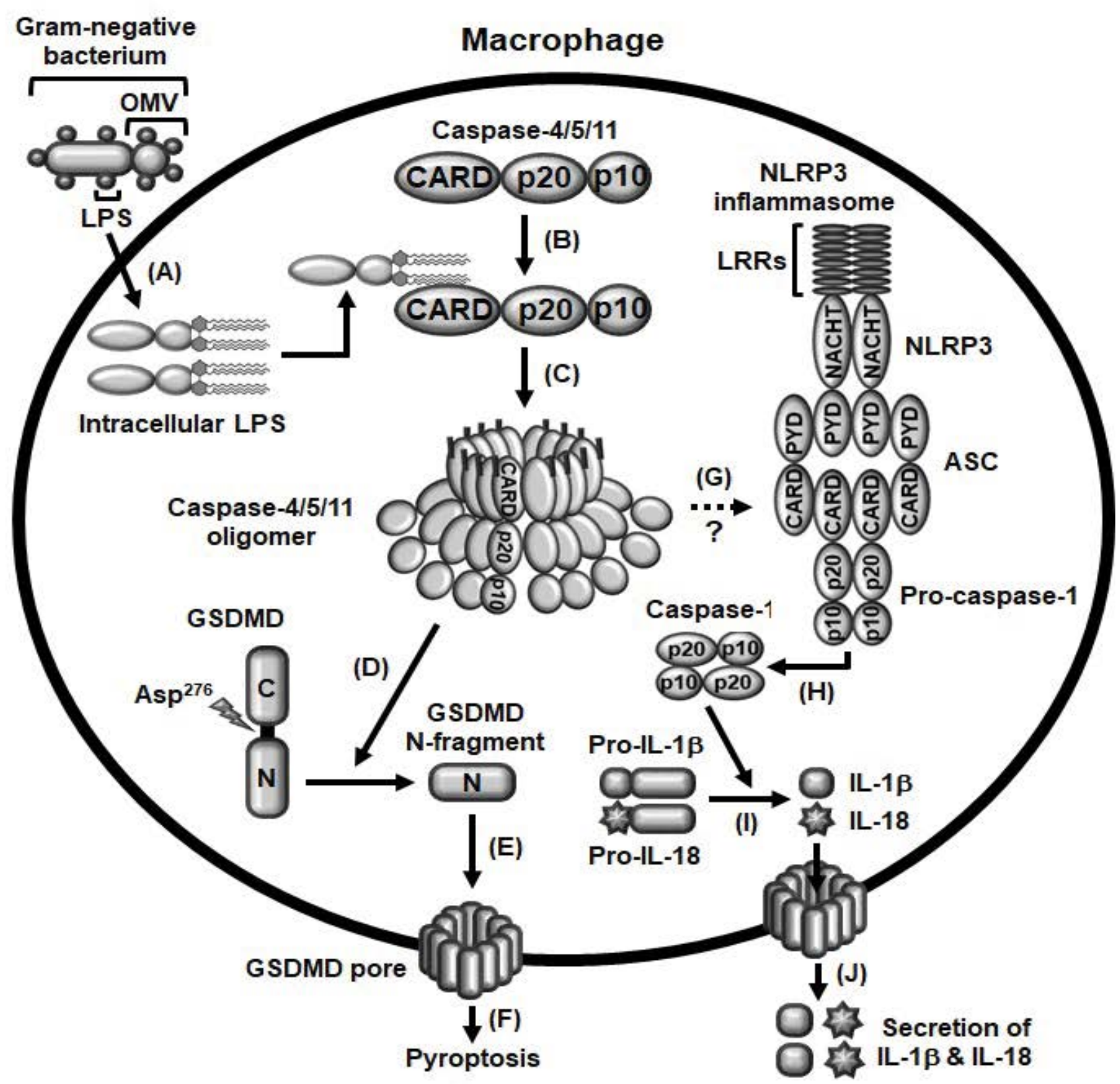

Figure 2: Schematic summary presenting the activation of caspase-4/5/11 non-canonical inflammasomes A) OMVs generated from gram-negative bacteria enter macrophages and LPS is released from OMVs; B) Intracellular LPS directly interacts with caspase-4/5/11 to produce LPS-caspase-4/5/11 complexes; C) These LPS-caspase-4/5/11 complexes are oligomerized, and the caspase-4/5/11 non-canonical inflammasomes are subsequently activated; D) Caspase-4/5/11 non-canonical inflammasomes cleave GSDMD at Asp276 residue to produce GSDMD N-terminal fragments; E) These GSDMD N-terminal fragments move to the membrane and generate GSDMD pores; F) GSDMD pores induce pyroptosis of macrophages; G) Caspase-4/5/11 non-canonical inflammasomes also activate NLRP3 canonical inflammasome with unknown mechanism; $\mathrm{H}$ ) NLRP3 inflammasome activation subsequently induces the proteolytic activation of pro-caspase-1; I) Active caspase-1 also induces the proteolytic maturation of pro-inflammatory cytokines, pro-IL-1 $\beta$ and pro-IL-18; J) Leading to the secretion of these mature and biologically active IL-1 $\beta$ and IL-18 through GSDMD pores.

OMV: Outer membrane vesicle; LPS: Lipopolysaccharide; Caspase: Cysteine-aspartic protease; GSDMD: Gasdermin D; N: GSDMD N-terminal fragment; C: GSDMD C-terminal fragment; CARD: Caspase recruit domain; PYD: Pyrin domain; NACHT: Nucleotide-binding and oligomerization domain; IL: Interleukin

?: Unknown mechanism.

of caspase-11 non-canonical inflammasome during inflammatory responses need to be investigated.

In conclusion, given the evidence from the previous studies, caspase-11 non-canonical inflammasome plays a pivotal role to induce inflammatory responses in macrophages. Furthermore, useful technologies to selectively target caspase-11 non-canonical inflammasome signa- ling, including CRISPR/Cas9, small interference RNA, and cell-penetrating antibody [29] could be a promising strategies to prevent and treat a variety of human infectious and inflammatory/autoimmune diseases.

\section{References}

1. Janeway CA Jr, R Medzhitov (2002) Innate immune recognition. Annu Rev Immunol 20: 197-216. 
2. Yi Young-Su (2016) Folate receptor-targeted diagnostics and therapeutics for inflammatory diseases. Immune Netw 16: $337-343$.

3. Kugelberg E (2014) Pattern recognition receptors: Curbing gut inflammation. Nat Rev Immunol 14: 583.

4. Yi YS, Son YJ, Ryou C, Sung GH, Kim JH, et al. (2014) Functional roles of Syk in macrophage-mediated inflammatory responses. Mediators Inflamm.

5. Yang Y, Kim SC, Yu T, Yi YS, Rhee MH, et al. (2014) Functional roles of p38 mitogen-activated protein kinase in macrophage-mediated inflammatory responses. Mediators Inflamm.

6. Yu T, Yi YS, Yang Y, Oh J, Jeong D, et al. (2012) The pivotal role of TBK1 in inflammatory responses mediated by macrophages. Mediators Inflamm.

7. Broz P, VM Dixit (2016) Inflammasomes: Mechanism of assembly, regulation and signalling. Nat Rev Immunol 16: 407-420.

8. Chen G, Shaw MH, Kim YG, Nunez G (2009) NOD-like receptors: Role in innate immunity and inflammatory disease. Annu Rev Pathol 4: 365-398.

9. Man SM, R Karki, TD Kanneganti (2017) Molecular mechanisms and functions of pyroptosis, inflammatory caspases and inflammasomes in infectious diseases. Immunol Rev 277: 61-75.

10. Yi YS (2017) Caspase-11 non-canonical inflammasome: A critical sensor of intracellular lipopolysaccharide in macrophage-mediated inflammatory responses. Immunology 152: 207-217.

11. Yi YS (2018) Role of inflammasomes in inflammatory autoimmune rheumatic diseases. Korean $\mathrm{J}$ Physiol Pharmacol 22: 1-15.

12. Ding JF (2017) Shao SnapShot: The noncanonical inflammasome. Cell 168: 544-544.e1.

13. Yi YS (2018) Regulatory roles of flavonoids on inflammasome activation during inflammatory responses. Mol Nutr Food Res 62: e1800147.

14. Man SM, R Karki, TD Kanneganti (2016) AIM2 inflammasome in infection, cancer, and autoimmunity: Role in DNA sensing, inflammation, and innate immunity. Eur $\mathrm{J}$ Immunol 46: 269-280.

15. Lugrin J, F Martinon (2018) The AIM2 inflammasome: Sensor of pathogens and cellular perturbations. Immunol Rev 281: 99-114.

16. Elena Vigano, Catherine Emma Diamond, Roberto Spreafico, Akhila Balachander, Radoslaw M Sobota, et al.
(2015) Human caspase- 4 and caspase- 5 regulate the onestep non-canonical inflammasome activation in monocytes. Nat Commun 6: 8761.

17. Casson CN, Yu J, Reyes VM, Taschuk FO, Yadav A, et al. (2015) Human caspase-4 mediates noncanonical inflammasome activation against gram-negative bacterial pathogens. Proc Natl Acad Sci U S A 112: 6688-6693.

18. Shi J, Zhao Y, Wang Y, Gao W, Ding J, et al. (2014) Inflammatory caspases are innate immune receptors for intracellular LPS. Nature 514: 187-192.

19. Kayagaki N, Warming S, Lamkanfi M, Vande Walle L, Louie $S$, et al. (2011) Non-canonical inflammasome activation targets caspase-11. Nature 479: 117-121.

20. Rathinam VA, Vanaja SK, Waggoner L, Sokolovska A, Becker C, et al. (2012) TRIF licenses caspase-11dependent NLRP3 inflammasome activation by gramnegative bacteria. Cell 150: 606-619.

21. Hagar JA, Powell DA, Aachoui Y, Ernst RK, Miao EA, et al. (2013) Cytoplasmic LPS activates caspase-11: Implications in TLR4-independent endotoxic shock. Science 341: 12501253.

22. Man SM, Karki R, Malireddi RK, Neale G, Vogel P, et al. (2015) The transcription factor IRF1 and guanylate-binding proteins target activation of the AIM2 inflammasome by Francisella infection. Nat Immunol 16: 467-475.

23. Kayagaki N, Wong MT, Stowe IB, Ramani SR, Gonzalez LC, et al. (2013) Noncanonical inflammasome activation by intracellular LPS independent of TLR4. Science 341: 12461249.

24. Mariathasan S (2007) ASC, Ipaf and Cryopyrin/Nalp3: Bona fide intracellular adapters of the caspase-1 inflammasome. Microbes Infect 9: 664-671.

25. Lamkanfi M, VM Dixit (2014) Mechanisms and functions of inflammasomes. Cell 157: 1013-1022.

26. Xiao Y, W Xu, W Su (2018) NLRP3 inflammasome: A likely target for the treatment of allergic diseases. Clin Exp Allergy 48: 1080-1091.

27. Shao BZ, Q Cao, C Liu (2018) Targeting nlrp3 inflammasome in the treatment of cns diseases. Front Mol Neurosci 11: 320.

28. Pavillard LE, Marin-Aguilar F, Bullon P, Cordero MD (2018) Cardiovascular diseases, NLRP3 inflammasome, and western dietary patterns. Pharmacol Res 131: 44-50.

29. Shin SM, Choi DK, Jung K, Bae J, Kim JS, et al. (2017) Antibody targeting intracellular oncogenic Ras mutants exerts anti-tumour effects after systemic administration. Nat Commun 8: 15090. 